\title{
Fuzzy Product KM-Subalgebras and Some Related Properties
}

\author{
K. Kalaiarasi $\left(\mathbb{D},{ }^{1}\right.$ V. Manimozhi $\left(\mathbb{D},{ }^{1}\right.$ N. Kausar $\mathbb{D}^{2},{ }^{2}$ D. Pamucar $\left(\mathbb{D},^{3}\right.$ \\ S. Kousar $\mathbb{D}^{4}{ }^{4}$ and Y. U. Gaba $\mathbb{D}^{5,6,7}$ \\ ${ }^{1} P G$ and Research Department of Mathematics, Cauvery College for Women (Autonomous), \\ Trichy-18 Affiliated to Bharathidasan University, Trichy, India \\ ${ }^{2}$ Department of Mathematics, Faculty of Arts and Science, Yildiz Technical University, Esenler, 34210, Istanbul, Turkey \\ ${ }^{3}$ Department of Logistics, University of Defence in Belgrade, Belgrade, Serbia \\ ${ }^{4}$ Department of Mathematics and Statistics, International Islamic University, Islamabad, Pakistan \\ ${ }^{5}$ Quantum Leap Africa (QLA) AIMS Rwanda Center, Remera Sector KN 3, Kigali, Rwanda \\ ${ }^{6}$ Institut de Mathématiques et de Sciences Physiques (IMSP/UAC), Laboratoire de Topologie Fondamentale, \\ Computationnelle et leurs Applications (Lab-ToFoCApp), BP 613, Porto-Novo, Benin \\ ${ }^{7}$ African Center for Advanced Studies, P.O. Box 4477, Yaounde, Cameroon
}

Correspondence should be addressed to Y. U. Gaba; yaeulrich.gaba@gmail.com

Received 8 July 2021; Accepted 15 September 2021; Published 6 October 2021

Academic Editor: Borna Abramović

Copyright (C) 2021 K. Kalaiarasi et al. This is an open access article distributed under the Creative Commons Attribution License, which permits unrestricted use, distribution, and reproduction in any medium, provided the original work is properly cited.

The concept of KM-algebras has been originated in 2019. KM-algebra is a generalization of some of the B-algebras such as BCK, $\mathrm{BCI}, \mathrm{BCH}, \mathrm{BE}$, and $\mathrm{BV}$ and also d-algebras. $\mathrm{KM}$-algebra serves two purposes in mathematics and computer science as follows: a tool for application in both fields and a strategy for creating the foundations. On the fuzziness of KM-algebras, an innovative perspective on fuzzy product KM-algebras as well as some related features is offered. Moreover, the notion of KMM-ideals is described and also initiated the concept of the KM-Cartesian product of fuzzy KM-algebras, and related outcomes are examined. Some of the innovative results in fuzzy KMM-ideals and KM-Cartesian product of fuzzy KM-subalgebras are analyzed, and some are as follows: arbitrary intersection of fuzzy KMM-ideals is again a fuzzy KMM-ideal, order reversing holds true in every KMMideal, every fuzzy KM-subalgebra is a fuzzy KMM-ideal, and KM-Cartesian product of two fuzzy KM-subalgebras is again a fuzzy KM-subalgebra.

\section{Introduction}

Ansari et al. [1, 2], Hong et al. [3], Qing-ping Hu [4], and Hwang et al. [5] researched a new class of algebra called $\mathrm{BCH}$-algebras and analyzed that the class of BCI-algebras is a proper subclass of $\mathrm{BCH}$-algebras. BCK-algebra was first introduced in 1966, thanks to the mathematicians Imai and Iséki [6] and has since been used in a variety of fields in mathematics, such as Topology, Probability theory, Functional Analysis, and Group theory. BCI-algebras are a generalization of BCK-algebras and were introduced by Iséki [7] in 1966.

Kalaiarasi et al. [8] introduced fuzzy sets in KM-algebras. Kim and Kim $[9,10]$ introduced the idea of BG-algebras which is a speculation of B-algebras. In 2007, Kim and Kim
[11] introduced another generalized BCK-algebras, namely, BE-algebras. Till now, so many B-algebras are developed by the researchers; refer [12-14]. In 2018, Mostafa and Cagman $[15,16]$ developed the concept of fuzzy dot structure in hyper KU-ideals. After BCK/BCI-algebras were introduced, many researchers developed algebraic structures which are the generalization of BCK/BCI-algebras. In 1999, Neggers and Kim [17-19] proposed a new class of algebraic structure, $\mathrm{d}$-algebra, which is a generalization of $\mathrm{BCK} / \mathrm{BCI} / \mathrm{B}$-algebras. In 2010, Saeid [20] presented fuzzy dot BCK/BCI-algebras. And in 2017, BI-algebras had been developed by Saei et al. [21]. In 2007, BF-algebras were developed by Walendziak. In 2021, Selvam and Priya [22] developed the idea of fuzzy dot $\mathrm{Z}$-ideals of Z-algebras by applying the idea of fuzzification of sets to Z-algebras. In 2014, Senapati et al. [23] presented 
fuzzy dot BG-algebras. In 2014, Senapati et al. [24] developed fuzzy dot subalgebras and ideals of B-algebras. In 2007, BFalgebras were developed by Walendziak [25] and all these algebras are generalizations of BCK/BCI-algebras.

One of the distinct parts of the set theories is fuzzy set and crisp set. In which, fuzzy set allows the elements to be included partially in the set whereas in a crisp set, the elements either belong to the set or not. Zadeh [26] introduced the concept of fuzzy sets in 1965 . Nowadays, there has been a vast development in fuzzy set. Many research works in various fields have been fuzzified. Many researchers who are working in the field of algebraic structures in mathematics have applied fuzzy sets, and the researchers are doing a tremendous job on this. The ideal hypothesis plays a crucial role in the ongoing advancement of $\mathrm{KM}$-algebras (KM named after Kalaiarasi and Mani). Fuzzy product subalgebras are fascinating logarithmic constructions and follow many intriguing outcomes. Additionally, fuzzy ideals are arrived at themes in any logarithmic design. Fuzzy product goals are helpful in numerical designs. Supposedly, no works are accessible on fuzzy product subalgebras of KM-algebras. The old style algebras are utilized to plan consistent circuits in PCs. Consequently, we persuaded to foster these hypotheses for KM-algebras.

Set theory supports one of the motivations. Among the several operations in set theory, there are three most vital and essential operations. The union, intersection, and set difference of two sets are performed using these fundamental operations. We get the concept of Boolean algebra if we regard these three operations and their qualities as a development of those three operations and their properties. The notion of Distributive Lattice is obtained by considering the union and intersection as a general algebra. Furthermore, we get the notions of an upper semilattice and a lower semilattice when we explore the concepts of union and intersection. Until now, properties of the set union, set intersection, set difference, and the set symmetric difference have been used to introduce numerous algebras. KM-algebras are one of the different algebra, highlighting the properties of the set theory such as the set union, the set intersection, and the set symmetric difference. The class of KM-algebras consists of a combination of four axioms of which two is from BCIalgebras, one from $\mathrm{B}$-algebras and in addition another axiom has been introduced. KM-algebra is a new class of algebra, and it motivates the algebraist to establish more interesting results and uses.

To do so, the rest of the article is organized as follows. In section 2, definition of KM-algebra, KMM-ideal, and fuzzy $\mathrm{KM}$-subalgebras with some examples has been given, and in section 3, we characterized fuzzy product subalgebras of KMalgebras and explored a great deal of properties. In section 4, definition of fuzzy KMM-ideal with examples and some of its properties has been discussed. In section 5 , a very interesting topic KM-Cartesian product of KM-algebras with some theorems has been presented. A reiteration of every single required definition and properties is given in the rest of this article.

Throughout this research paper, we consider ' $\bullet$ ' as the binary operation of KM-algebras.

\section{Preliminaries}

Some preliminary definitions for KM-algebra, KMM-ideal, and fuzzy KM-subalgebras have been given in this section.

Definition 1 (see [8]). Let $\mathrm{K}$ be a set, together with a binary operation ' $\bullet$ ' and a constant ' 0 '. Then, $K=(K, 0)$ is called a KM-algebra [8] satisfying the following conditions:

(i) (KM1) $k \bullet m=0$ if $k=m$

(ii) (KM2) $k \bullet m=k$ if $m=0$

(iii) $(\mathrm{KM} 3)(k \bullet m) \bullet n=n \bullet(k \bullet m)$

(iv) (KM4) $k \bullet m=0$ and $m \bullet k=0$ imply $k=m$, for all $\bullet k, m, n \in K$

Example 1. Let $M=\left\{m_{00}, m_{01}, m_{02}\right\}$ be a set with the binary operation - and a constant $m_{00}$ with Table 1 .

Here, in this example, the constant ' 0 ' in the definition given is ' $m_{00}$ '.

(i) (KM1) $k \bullet m=m_{00}$ if $k=m$. Here, $m_{00}$ appears in the diagonal from upper left to lower right. The first condition KM1 is true in all the three cases.

(ii) (KM2) $k \bullet m=k$ if $m=m_{00}$. From the first row of Table 1, KM2 is true.

(iii) (KM3) $(k \bullet m) \bullet n=n \bullet(k \bullet m)$, for all $k, m, n \in K$. From Table 2, the condition for (KM3) is satisfied.

(iv) (KM4) $k \bullet m=m_{00}$ and $m \bullet k=m_{00}$ imply $k=\mathrm{m}$, for all $k, m, n \in K$.

Therefore, the condition KM4 is true. $\left(\mathrm{M}, \bullet, m_{00}\right)$ is a KM-algebra.

Definition 2 (see [8]). Let $\mathrm{K}$ be a KM-algebra and $I_{d}$ be a subset of $\mathrm{K}$ which is nonempty. Then, we call $I_{d}$ as the $\mathrm{KMM}$-ideal [8] of $\mathrm{K}$ if the following conditions are satisfied:

(1) $0 \in I_{d}$

(2) $k \bullet m \in I_{d}$ and $m \in I_{d}$ imply $k \in I_{d}$ or all $k, m \in K$

Example 2. For Example 1, the KMM-ideals of $\mathrm{M}$ are $\left\{m_{00}, m_{01}\right\},\left\{m_{00}, m_{01}, m_{02}\right\}$.

Definition 3. Let B be a fuzzy set in a KM-algebra K. Then, B is said to be a fuzzy KM-subalgebra [8] of $\mathrm{K}$ if the following condition holds: $\lambda(k \bullet m) \geq \wedge\{\lambda(k), \lambda(m)\}$, for all $k, m \in B$. Here, $\lambda$ is the function on $\mathrm{K}$ such that $\lambda(k) \leq 1$ for all $k \in B$.

Example 3. In Example 1, define a fuzzy set $\lambda: B \longrightarrow[0,1] \lambda\left(m_{00}\right)=0.5, \lambda\left(m_{01}\right)=0.3, \lambda\left(m_{02}\right)=0.1$.

From Table 3, obviously the condition for fuzzy KMsubalgebras is satisfied. Thus, B satisfies the conditions of the fuzzy KM-subalgebras of M.

\section{Fuzzy Product KM-Subalgebra}

We introduced fuzzy product KM-algebra with some examples, and interesting results are investigated. 
TABLE 1: Binary operation.

\begin{tabular}{llll}
\hline$\bullet$ & $m_{00}$ & $m_{01}$ & $m_{02}$ \\
\hline$m_{00}$ & $m_{00}$ & $m_{01}$ & $m_{02}$ \\
$m_{01}$ & $m_{01}$ & $m_{00}$ & $m_{02}$ \\
$m_{02}$ & $m_{02}$ & $m_{02}$ & $m_{00}$ \\
\hline
\end{tabular}

TABLE 2: $(\mathbf{k} \bullet \mathbf{m}) \bullet \boldsymbol{n}=\boldsymbol{n} \bullet(\mathbf{k} \bullet \mathbf{m})$.

\begin{tabular}{lcc}
\hline$(\mathbf{k}, \mathbf{m}, \mathbf{n})$ & $(\mathbf{k} \bullet \mathbf{m}) \bullet \mathbf{n}$ & $(\mathbf{k} \bullet \mathbf{n}) \bullet \mathbf{m}$ \\
\hline$\left(\mathbf{m}_{00}, \mathbf{m}_{00}, \mathbf{m}_{00}\right)$ & $m_{00}$ & $m_{00}$ \\
$\left(\mathbf{m}_{00}, \mathbf{m}_{00}, \mathbf{m}_{01}\right)$ & $m_{01}$ & $m_{01}$ \\
$\left(\mathbf{m}_{00}, \mathbf{m}_{00}, \mathbf{m}_{02}\right)$ & $m_{02}$ & $m_{02}$ \\
$\left(\mathbf{m}_{00}, \mathbf{m}_{01}, \mathbf{m}_{00}\right)$ & $m_{01}$ & $m_{01}$ \\
$\left(\mathbf{m}_{00}, \mathbf{m}_{01}, \mathbf{m}_{01}\right)$ & $m_{00}$ & $m_{00}$ \\
$\left(\mathbf{m}_{00}, \mathbf{m}_{01}, \mathbf{m}_{02}\right)$ & $m_{02}$ & $m_{02}$ \\
$\left(\mathbf{m}_{00}, \mathbf{m}_{02}, \mathbf{m}_{00}\right)$ & $m_{02}$ & $m_{02}$ \\
$\left(\mathbf{m}_{0 ?}, \mathbf{m}_{02}, \mathbf{m}_{01}\right)$ & $m_{02}$ & $m_{02}$ \\
$\left(\mathbf{m}_{00}, \mathbf{m}_{02}, \mathbf{m}_{02}\right)$ & $m_{00}$ & $m_{00}$ \\
$\left(\mathbf{m}_{01}, \mathbf{m}_{00}, \mathbf{m}_{00}\right)$ & $m_{01}$ & $m_{01}$ \\
$\left(\mathbf{m}_{01}, \mathbf{m}_{00}, \mathbf{m}_{01}\right)$ & $m_{00}$ & $m_{00}$ \\
$\left(\mathbf{m}_{01}, \mathbf{m}_{00}, \mathbf{m}_{02}\right)$ & $m_{02}$ & $m_{02}$ \\
$\left(\mathbf{m}_{01}, \mathbf{m}_{01}, \mathbf{m}_{00}\right)$ & $m_{00}$ & $m_{00}$ \\
$\left(\mathbf{m}_{01}, \mathbf{m}_{01}, \mathbf{m}_{01}\right)$ & $m_{00}$ & $m_{00}$ \\
$\left(\mathbf{m}_{01}, \mathbf{m}_{01}, \mathbf{m}_{02}\right)$ & $m_{02}$ & $m_{02}$ \\
$\left(\mathbf{m}_{01}, \mathbf{m}_{02}, \mathbf{m}_{00}\right)$ & $m_{02}$ & $m_{02}$ \\
$\left(\mathbf{m}_{01}, \mathbf{m}_{02}, \mathbf{m}_{01}\right)$ & $m_{02}$ & $m_{02}$ \\
$\left(\mathbf{m}_{01}, \mathbf{m}_{02}, \mathbf{m}_{02}\right)$ & $m_{00}$ & $m_{00}$ \\
$\left(\mathbf{m}_{02}, \mathbf{m}_{00}, \mathbf{m}_{00}\right)$ & $m_{02}$ \\
$\left(\mathbf{m}_{02}, \mathbf{m}_{00}, \mathbf{m}_{01}\right)$ & $m_{02}$ & $m_{02}$ \\
$\left(\mathbf{m}_{02}, \mathbf{m}_{00}, \mathbf{m}_{02}\right)$ & $m_{00}$ & $m_{00}$ \\
$\left(\mathbf{m}_{02}, \mathbf{m}_{01}, \mathbf{m}_{00}\right)$ & $m_{02}$ & $m_{02}$ \\
$\left(\mathbf{m}_{02}, \mathbf{m}_{01}, \mathbf{m}_{01}\right)$ & $m_{02}$ & $m_{02}$ \\
$\left(\mathbf{m}_{02}, \mathbf{m}_{01}, \mathbf{m}_{02}\right)$ & $m_{00}$ & $m_{00}$ \\
$\left(\mathbf{m}_{02}, \mathbf{m}_{02}, \mathbf{m}_{00}\right)$ & $m_{00}$ & $m_{00}$ \\
$\left(\mathbf{m}_{02}, \mathbf{m}_{02}, \mathbf{m}_{01}\right)$ & $m_{01}$ & $m_{01}$ \\
$\left(\mathbf{m}_{02}, \mathbf{m}_{02}, \mathbf{m}_{02}\right)$ & $m_{00}$ & $m_{00}$ \\
\hline & &
\end{tabular}

TABLE 3: $\lambda(k \bullet m) \geq \wedge\{\lambda(k), \lambda(m)\}$.

\begin{tabular}{lcc}
\hline$k \bullet m$ & $\lambda(k \bullet m)$ & $\wedge\{\lambda(k), \lambda(m)\}$ \\
\hline$m_{00} \bullet m_{00}=m_{00}$ & 0.5 & 0.5 \\
$m_{00} \bullet m_{01}=m_{01}$ & 0.3 & 0.3 \\
$m_{00} \bullet m_{02}=m_{02}$ & 0.1 & 0.1 \\
$m_{01} \bullet m_{00}=m_{01}$ & 0.3 & 0.3 \\
$m_{01} \bullet m_{01}=m_{00}$ & 0.5 & 0.3 \\
$m_{01} \bullet m_{02}=m_{02}$ & 0.1 & 0.1 \\
$m_{02} \bullet m_{00}=m_{02}$ & 0.1 & 0.1 \\
$m_{02} \bullet m_{01}=m_{02}$ & 0.1 & 0.1 \\
$m_{02} \bullet m_{02}=m_{00}$ & 0.5 & 0.1 \\
\hline
\end{tabular}

Definition 4. A fuzzy set $\mathrm{B}$ subset of $\mathrm{K}$ is called a fuzzy product KM-subalgebra of a KM-algebra $\mathrm{K}$ if the following condition is satisfied: $\lambda_{P_{\mathrm{dt}}}(k \bullet m) \geq \lambda_{P_{\mathrm{dt}}}(k) p_{\mathrm{d} t} \lambda_{P_{\mathrm{dt}}}(m)$, for all $k, m \in B$ and $\lambda_{P_{d t}}$, the functions on $\mathrm{K}$ such that $0 \leq \lambda_{P_{\mathrm{dt}}}(k) \leq 1$ for all $k \in B$ where $P_{d t}$ denotes the multiplication.

\section{Example 4}

(1) In Example 1, we define $\lambda_{p_{\mathrm{dt}}}\left(m_{00}\right)=0.3$, $\lambda_{p_{\mathrm{dt}}}\left(m_{01}\right)=0.5$, and $\lambda_{p_{\mathrm{d} t}}\left(m_{02}\right)=0.4$.
In Table 4 , the value for $\lambda_{p_{\mathrm{dt}}}(k) P_{\mathrm{dt}} \lambda_{p_{\mathrm{dt}}}(m)$ is given. The condition of the fuzzy product KM-algebra is verified from Table 5.

Therefore, $\quad \lambda_{p_{\mathrm{dt}}}(k \bullet m) \geq \lambda_{p_{\mathrm{dt}}}(k) p_{\mathrm{d} t} \lambda_{p_{\mathrm{dt}}}(m), \quad$ for all $k, m \in B$. Thus, $\mathrm{M}$ satisfies the condition and hence a fuzzy product KM-subalgebra. If $B=\left(\begin{array}{lll}0.09 & 0.15 & 0.12 \\ 0.15 & 0.25 & 0.20 \\ 0.12 & 0.20 & 0.16\end{array}\right)$, then $B$ is a symmetric fuzzy matrix, and the elements of the matrix belong to fuzzy unit interval $[0,1]$.

(2) Let us consider the KM-algebra $N=\left\{n_{00}, n_{01}, n_{02}, n_{03}\right\}$, having the following Cayley table. Here, the constant ' 0 ' is ' $n_{00}$.' From Table 6, clearly $\left(N, \bullet, n_{00}\right)$ is a KM-algebra.

$\left(\mathrm{N}, \bullet, n_{00}\right)$ is a KM-algebra.

Define $\quad \lambda_{P_{\mathrm{dt}}}\left(n_{01}\right)=\lambda_{P_{\mathrm{dt}}}\left(n_{02}\right)=0.3 \quad$ and $\lambda_{P_{\mathrm{dt}}}\left(n_{03}\right)=0.7$.

In Table 7, product of $\lambda_{P_{\mathrm{dt}}}(k)$ and $\lambda_{P_{\mathrm{dt}}}(m)$ has be given.

The condition has been checked.

In Table 8, the condition for fuzzy product KMsubalgebras is verified. Then, $\mathrm{N}$ satisfies the conditions of the fuzzy product KM-subalgebras.

(3) Define $\lambda_{P_{\mathrm{d} t}}(k)=0.73$ and $\lambda_{P_{\mathrm{dt}}}(m)=0.023$ and

$$
\lambda_{P_{\mathrm{dt}}}(k \cdot m)= \begin{cases}0, & \text { if } k \leq m, \\ 0.6, & \text { otherwise. }\end{cases}
$$

It is clear that $\lambda_{P_{\mathrm{dt}}}(k \cdot m) \geq \lambda_{P_{\mathrm{d} t}}(k) P_{\mathrm{d} t}$ $\lambda_{P_{\mathrm{d} t}}(m)$, for all $k, m \in K$.

Therefore, $\mathrm{K}$ is a fuzzy product $\mathrm{KM}$-subalgebra.

(4) Also, we shall define

$$
\begin{aligned}
\lambda_{P_{\mathrm{dt}}}(k) & =0.3 \text { and } \lambda_{P_{\mathrm{dt}}}(m)=0.32, \\
\lambda_{P_{\mathrm{dt}}}(k \cdot m) & = \begin{cases}0, & \text { if } k \leq m, \\
0.6, & \text { otherwise. }\end{cases}
\end{aligned}
$$

This does not satisfy $\lambda_{P_{\mathrm{dt}}}(k \cdot m) \geq \lambda_{P_{\mathrm{dt}}}(k) P_{\mathrm{d} t} \lambda_{P_{\mathrm{d} t}}(m)$, for all $k, m \in K$. Therefore, $\mathrm{K}$ is not a fuzzy product $\mathrm{KM}$ subalgebra.

Remark 1. Every fuzzy KM-subalgebra is fuzzy product KM-subalgebras of a KM-algebra, but the converse need not be true. Example $4 \lambda_{P_{\mathrm{dt}}}\left(m_{01} \bullet m_{01}\right)=\lambda_{p_{\mathrm{dt}}}\left(m_{00}\right)=0.3<$ $\lambda_{p_{\mathrm{d} t}}\left(m_{01}\right)=\mathrm{V}\left\{\lambda_{p_{\mathrm{d} t}}\left(m_{01}\right), \lambda_{p_{\mathrm{d} t}}\left(m_{01}\right)\right\}$.

Theorem 1. If $B$ is a fuzzy product KM-subalgebra with a membership function $\lambda_{p_{d t}}$ of a KM-algebra $K$, then $\lambda_{p_{d t}}(0) \geq\left[\lambda_{p_{d t}}(k)\right]^{n}$, for all $a \in K$ and $n \geq 2$ and $n$ is a positive integer.

Proof. By mathematical induction on $n$, this theorem can be proved. For $n=2$, 
Table 4: Product $P_{\mathrm{d} t}$.

\begin{tabular}{lccc}
\hline$P_{\mathrm{d} t}$ & $\lambda_{P_{\mathrm{d} t}}\left(m_{00}\right)$ & $\lambda_{P_{\mathrm{d} t}}\left(m_{01}\right)$ & $\lambda_{P_{\mathrm{d} t}}\left(m_{02}\right)$ \\
\hline$\lambda_{P_{\mathrm{d} t}}\left(m_{00}\right)$ & 0.09 & 0.15 & 0.12 \\
$\lambda_{P_{\mathrm{d} t}}\left(m_{01}\right)$ & 0.15 & 0.25 & 0.20 \\
$\lambda_{P_{\mathrm{d} t}}\left(m_{02}\right)$ & 0.12 & 0.20 & 0.16 \\
\hline
\end{tabular}

\begin{tabular}{lcc} 
TABLE $5: \lambda_{p_{\mathrm{dt}}}(k \bullet m) \geq \lambda_{p_{\mathrm{d} t}}(k) p_{\mathrm{d} t} \lambda_{p_{\mathrm{dt}}}(m)$. \\
\hline$k \bullet m$ & $\lambda_{p_{\mathrm{dt}}}(k \bullet m)$ & $\lambda_{p_{\mathrm{d} t}}(k) p_{\mathrm{d} t} \lambda_{p_{\mathrm{dt}}}(m)$ \\
\hline$m_{00} \bullet m_{00}=m_{00}$ & 0.3 & 0.09 \\
$m_{00} \bullet m_{01}=m_{01}$ & 0.5 & 0.15 \\
$m_{00} \bullet m_{02}=m_{02}$ & 0.4 & 0.12 \\
$m_{01} \bullet m_{00}=m_{01}$ & 0.5 & 0.15 \\
$m_{01} \bullet m_{01}=m_{00}$ & 0.3 & 0.25 \\
$m_{01} \bullet m_{02}=m_{02}$ & 0.4 & 0.20 \\
$m_{02} \bullet m_{00}=m_{02}$ & 0.4 & 0.12 \\
$m_{02} \bullet m_{01}=m_{02}$ & 0.4 & 0.20 \\
$m_{02} \bullet m_{02}=m_{00}$ & 0.3 & 0.16
\end{tabular}

TABLe 6: Operator.

\begin{tabular}{lllll}
\hline$\bullet$ & $n_{00}$ & $n_{01}$ & $n_{02}$ & $n_{03}$ \\
\hline$n_{00}$ & $n_{00}$ & $n_{01}$ & $n_{02}$ & $n_{03}$ \\
$n_{01}$ & $n_{01}$ & $n_{00}$ & $n_{03}$ & $n_{02}$ \\
$n_{02}$ & $n_{02}$ & $n_{03}$ & $n_{00}$ & $n_{01}$ \\
$n_{03}$ & $n_{03}$ & $n_{02}$ & $n_{01}$ & $n_{00}$ \\
\hline
\end{tabular}

Table 7: Product $P_{\mathrm{d} t}$.

\begin{tabular}{lcccc}
\hline$P_{\mathrm{d} t}$ & $\lambda_{P_{\mathrm{d} t}}\left(n_{00}\right)$ & $\lambda_{P_{\mathrm{d} t}}\left(n_{01}\right)$ & $\lambda_{P_{\mathrm{d} t}}\left(n_{02}\right)$ & $\lambda_{P_{\mathrm{d} t}}\left(n_{03}\right)$ \\
\hline$\lambda_{P_{\mathrm{d} t}}\left(n_{00}\right)$ & 0.81 & 0.27 & 0.27 & 0.63 \\
$\lambda_{P_{\mathrm{d} t}}\left(n_{01}\right)$ & 0.27 & 0.09 & 0.09 & 0.21 \\
$\lambda_{P_{\mathrm{d} t}}\left(n_{02}\right)$ & 0.27 & 0.09 & 0.09 & 0.21 \\
$\lambda_{P_{\mathrm{d} t}}\left(n_{03}\right)$ & 0.63 & 0.21 & 0.21 & 0.49 \\
\hline
\end{tabular}

TABLE 8: $\lambda_{P_{\mathrm{d} t}}(k \bullet m) \geq \lambda_{P_{\mathrm{d} t}}(k) p_{\mathrm{d} t} \lambda_{P_{\mathrm{d} t}}(m)$.

\begin{tabular}{lcc}
\hline$k \bullet m$ & $\lambda_{P_{d t}}(k \bullet m)$ & $\lambda_{P_{d t}}(k) p_{d t} \lambda_{P_{d t}}(m)$ \\
\hline$n_{00} \bullet n_{00}=n_{00}$ & 0.9 & 0.81 \\
$n_{00} \bullet n_{01}=n_{01}$ & 0.3 & 0.27 \\
$n_{00} \bullet n_{02}=n_{02}$ & 0.3 & 0.27 \\
$n_{00} \bullet n_{03}=n_{03}$ & 0.7 & 0.63 \\
$n_{01} \bullet n_{00}=n_{01}$ & 0.3 & 0.27 \\
$n_{01} \bullet n_{01}=n_{00}$ & 0.9 & 0.09 \\
$n_{01} \bullet n_{02}=n_{03}$ & 0.7 & 0.09 \\
$n_{01} \bullet n_{03}=n_{02}$ & 0.3 & 0.21 \\
$n_{02} \bullet n_{00}=n_{02}$ & 0.3 & 0.27 \\
$n_{02} \bullet n_{01}=n_{03}$ & 0.7 & 0.09 \\
$n_{02} \bullet n_{02}=n_{00}$ & 0.9 & 0.09 \\
$n_{02} \bullet n_{03}=n_{01}$ & 0.3 & 0.21 \\
$n_{03} \bullet n_{00}=n_{03}$ & 0.7 & 0.63 \\
$n_{03} \bullet n_{01}=n_{02}$ & 0.3 & 0.21 \\
$n_{03} \bullet n_{02}=n_{01}$ & 0.3 & 0.21 \\
$n_{03} \bullet n_{03}=n_{00}$ & 0.9 & 0.49 \\
\hline
\end{tabular}

$$
\begin{aligned}
\lambda_{p_{\mathrm{d} t}}(0) & \left.=\lambda_{p_{\mathrm{d} t}}(k \bullet k)\right) \\
& \geq \lambda_{p_{\mathrm{d} t}}(k) p \mathrm{~d} \lambda_{p_{\mathrm{d} t}}(k) \\
& =\left(\lambda_{p_{\mathrm{d} t}}(k)\right)^{2} .
\end{aligned}
$$

Therefore, for $n=2$, the result is true. Assume that the result is true for $n=r>2$. That is, $\lambda_{p_{\mathrm{d} t}}(0) \geq\left[\lambda_{p_{\mathrm{d} t}}(k)\right]^{r}$, for all $r \geq 2$. Now, to prove for $n=r+1$, the result is true.

$$
\begin{aligned}
\lambda_{P_{\mathrm{d} t}}(0) & \geq\left(\lambda_{P_{\mathrm{d} t}}(k)\right)^{r}-\lambda_{P_{\mathrm{d} t}}(k) p_{\mathrm{d} t} \lambda_{P_{\mathrm{d} t}}(0) \\
& \geq\left(\lambda_{P_{\mathrm{d} t}}(a)\right)^{r+1} \lambda_{P_{\mathrm{d} t}}(k \bullet 0) \\
& \geq\left(\lambda_{P_{\mathrm{d} t}}(k)\right)^{r+1} \lambda_{P_{\mathrm{d} t}}(0) \\
& \geq\left(\lambda_{P_{\mathrm{d} t}}(k)\right)^{r+1} .
\end{aligned}
$$

For every $n \geq 2$, the result is true.

Theorem 2. Let B be a fuzzy product KM-subalgebra with the membership function $\lambda_{P_{d t}}$ of a KM-algebra $K$. If there exists a sequence $\left\{u_{m}\right\}$ in $K$ such that $\left(\left[\lambda_{P_{d t}}\left(u_{m}\right)\right]^{n}\right) \longrightarrow u$, then $u \leq \lambda_{P_{d t}}(0) \leq 1$.

Proof. By Theorem 1, $\lambda_{P_{\mathrm{d} t}}(0) \geq\left[\lambda_{P_{\mathrm{d} t}}(k)\right]^{n}$ for every $k \in K$ and $n \geq 2$, and $n$ is a positive integer.

$$
\begin{aligned}
& \lim _{m \longrightarrow \infty} \lambda_{P_{\mathrm{d} t}}(0) \geq \lim _{m \longrightarrow \infty}\left[\lambda_{P_{\mathrm{d} t}}\left(u_{m}\right)\right]^{n}=u \\
& \Longrightarrow \lim _{m \longrightarrow \infty} \lambda_{P_{\mathrm{d} t}}(0) \geq u \text {. since } 1 \geq \lambda_{P_{\mathrm{d} t}}(0) \\
& \Longrightarrow \lim _{m \longrightarrow \infty} \lambda_{P_{\mathrm{d} t}}(0) \geq u \text {. since } \lambda_{P_{\mathrm{d} t}}(0) \leq 1 \\
& \Longrightarrow u \leq \lim _{m \longrightarrow \infty} \lambda_{P_{\mathrm{d} t}}(0) \leq 1 .
\end{aligned}
$$

In particular, if the limit $u=1$, then $\lambda_{P_{\mathrm{d} t}}(0) \leq 1$. and $\lim _{m \longrightarrow \infty} \lambda_{P_{\mathrm{d} t}}(0) \geq \lim _{m \longrightarrow \infty}\left[\lambda_{P_{\mathrm{d} t}}\left(u_{m}\right)\right]^{n}=u=1$ imply that $\lambda_{P_{\mathrm{d} t}}(0)=1$.

The intersection of two fuzzy product KM-subalgebras is also a fuzzy product KM-subalgebra, and the result is proved in the following theorem.

Theorem 3. If $\left(S, \sigma_{P_{d t}}\right)$ and $\left(G, \rho_{P_{d t}}\right)$ are the fuzzy product $K M$-subalgebra of a KM-algebra $K$, then $\left(S \cap G, \sigma_{P_{d t}} \wedge \rho_{P_{d t}}\right)$.

Proof. Let $a \in S, b \in G$. Clearly $k, m \in K$. Now,

$$
\begin{aligned}
\left(\sigma_{P_{\mathrm{d} t}} \wedge \rho_{P_{\mathrm{d} t}}\right)(k \bullet m)= & \wedge\left\{\sigma_{P_{\mathrm{d} t}}(k \bullet m), \rho_{P_{\mathrm{d} t}}(k \bullet m)\right\} \\
& \geq \wedge\left\{\sigma_{P_{\mathrm{d} t}}(k) p \mathrm{~d} \sigma_{P_{\mathrm{d} t}}(m), \rho_{P_{\mathrm{d} t}}(k) p \mathrm{~d} \rho_{P_{\mathrm{d} t}}(m)\right\} \\
& \geq \wedge\left\{\sigma_{P_{\mathrm{d} t}}(k) \rho_{\mathrm{d} t}(k)\right\}, p_{\mathrm{d} t} \wedge\left\{\sigma_{P_{\mathrm{d} t}}(m), \rho_{P_{\mathrm{d} t}}(m)\right\} \\
= & \left(\sigma_{P_{\mathrm{d} t}} \wedge \rho_{\mathrm{d} t}\right)(k) \rho_{P_{\mathrm{d} t}}\left(\sigma_{P_{\mathrm{d} t}} \wedge \rho_{\mathrm{d} t}\right)(m) .
\end{aligned}
$$

Hence, the result is obtained. Also, if B is a fuzzy product KM-subalgebra with the membership functions $\lambda_{P_{\mathrm{d} t}}$ of a 
KM-algebra $\mathrm{K}$, for $i \in N$, then $\cap B_{i}$ is also a fuzzy product KM-subalgebra of $\mathrm{K}$.

Theorem 4. $B^{s}(s \geq 1)$ is fuzzy product KM-subalgebra of $K$. If $B$ is a fuzzy product KM-subalgebra of $K$, then $B^{s}(s \geq 1)$ is also a fuzzy product KM-subalgebra of $K$.

Proof. Let $k, m \in K . B^{s}(s \geq 1)$ is a fuzzy set of $\mathrm{K}$ defined by $\lambda_{P_{\mathrm{d} t}}(k)=\left[\lambda_{P_{\mathrm{d} t}}(k)\right]^{s}, s \geq 1$. Also, given $\mathrm{B}$ is a fuzzy product KM-subalgebra of $\mathrm{K}$.

Now, for $s \geq 1$,

$$
\begin{aligned}
\lambda_{P_{\mathrm{d} t}}(k \bullet m) & =\left[\lambda_{P_{\mathrm{d} t}}(k \bullet m)\right]^{s}, \\
& \geq\left[\lambda_{P_{\mathrm{d} t}}(k) p_{\mathrm{d} t} \lambda_{P_{\mathrm{d} t}}(m)\right]^{s}, \\
& =\left[\lambda_{P_{\mathrm{d} t}}(k)\right]^{s} p_{\mathrm{d} t}\left[\lambda_{P_{\mathrm{d} t}}(m)\right]^{s}, \\
& =\lambda_{P_{\mathrm{d} t}}(k) p_{\mathrm{d} t} \lambda_{P_{\mathrm{d} t}}(m) .
\end{aligned}
$$

Therefore, $\lambda_{P_{\mathrm{d} t}}(k \bullet m) \geq \lambda_{P_{\mathrm{d} t}}(k) p \mathrm{~d} \lambda_{P_{\mathrm{d} t}}(m)$.

Theorem 5. The set $M(X, 1)=\left\{k \in K / \lambda_{P_{d t}}(k)=1\right\}$ is either empty or is a KM-subalgebra of $K$, where $X$ is a fuzzy product KM-algebra.

Proof. Suppose $M(X, 1)$ is nonempty.

Let $k, m \in M(X, 1) \Longrightarrow \lambda_{P_{\mathrm{d} t}}(k)=1$ and $\lambda_{P_{\mathrm{d} t}}(m)=1$.

$$
\begin{aligned}
\lambda_{P_{\mathrm{d} t}}(k \bullet m) & \geq \lambda_{P_{\mathrm{d} t}}(k) p_{\mathrm{d} t} \lambda_{P_{\mathrm{d} t}}(m)=1 \\
& \Longrightarrow k \bullet m \in M(X, 1) .
\end{aligned}
$$

Therefore, $\mathrm{M}(\mathrm{X}, 1)$ is a KM-subalgebra.

Theorem 6. Let $g: U \longrightarrow V$ be an onto homomorphism of a $K M$-algebra $K$. If $B$ is a fuzzy product KM-subalgebra of $U$, then the image $g(B)$ of $B$ under $g$ is a fuzzy product KMsubalgebra of $V$.

Proof. Let $d_{11}, d_{12} \in V$. Let $C_{1}=g^{-1}\left(d_{11}\right)$ and $C_{2}$ $=g^{-1}\left(d_{12}\right)$ and $C_{12}=g^{-1}\left(d_{11} \bullet d_{12}\right)$.

Consider

$$
\begin{aligned}
& C_{1} \bullet C_{2}=\left\{c \bullet \frac{d}{c} \in U, d \in V\right\} \\
& =g^{-1}\left(d_{11} \bullet d_{12}\right) \in C_{12} \\
& C_{1} \bullet C_{2} \subseteq C_{12} \\
& g(B)(c \bullet d)=\operatorname{Sup}_{c \bullet d \in g^{-1}\left(d_{11} \bullet d_{12}\right)} g(c \bullet d) \\
& =\operatorname{Sup}_{c \bullet d \in C_{12}} g(c \bullet d) \\
& \geq \operatorname{Sup}_{c \bullet d \in C_{1} \bullet C_{2}} g(c \bullet d) \\
& \geq \operatorname{Sup}_{c \in C_{1}, d \in C_{2}} g(c \bullet d) \\
& \geq \operatorname{Sup}_{c \in C_{1}, d \in C_{2}} g(c) \bullet g(d) \text {. }
\end{aligned}
$$

Therefore, $[0,1] \times[0,1] \longrightarrow[0,1]$ is continuous; then, for any $\varepsilon>0$, there exists a $\delta>0$ such that $\tilde{c} \geq$ $\sup _{c \in C_{1}} g(c)-\delta$ and $\tilde{d} \geq \sup _{d \in C_{2}} g(d)-\delta$. Then, $\quad \tilde{c} \bullet \widetilde{d} \geq$ $\sup _{c \in C_{1}} g(c) p_{\mathrm{d} t} \sup _{d \in C_{2}} g(d)-\varepsilon$. Choose $c_{1} \in C_{1}$ and $c_{2} \in C_{2}$ such that

$$
\begin{aligned}
g\left(c_{1}\right) & \geq \sup _{c \in C_{1}} g(c)-\delta \text { and } g\left(c_{2}\right) \geq \sup _{d \in C_{2}} g(d)-\delta \\
g\left(c_{1}\right) p \mathrm{~d} g\left(c_{2}\right) & \geq \sup _{c \in C_{1}} g(c) p d \sup _{d \in C_{2}} g(d)-\varepsilon \\
g(B)(c \bullet d) & \geq \operatorname{Sup}_{c \in C_{1}, d \in C_{2}} g(c) \bullet g(d) \\
& \geq \operatorname{Sup}_{c \in C_{1}} g(c) p_{\mathrm{d} t} \operatorname{Sup}_{d \in C_{2}} g(d) \\
& =g(B)(c) p_{\mathrm{d} t} g(B)(d) .
\end{aligned}
$$

Hence, $\mathrm{g}(\mathrm{B})$ is a fuzzy product KM-subalgebra.

\section{Fuzzy KMM-Ideals}

A new notion of fuzzy KMM-ideal has been introduced with an example, and some related results have been investigated.

Definition 5. Let $\widetilde{F}$ be a fuzzy set in K. Then, we call $\widetilde{F}$ as a fuzzy KMM-ideal of $\mathrm{K}$ if the following inequality hold:

$(\mathrm{KMI} 1) \quad \widetilde{F}(0)=\widetilde{F}(k),(\mathrm{KMI} 2) \quad \widetilde{F}(k) \geq \wedge\{\widetilde{F}(k \bullet m)$, $\widetilde{F}(m)\}$, for all $k, m \in K$.

Example 5. Define $\widetilde{F}$ in $\mathrm{M}$ of Example 1 by $\widetilde{F}(k)=\left\{\begin{array}{ll}0.1 & k>0 \text { in } K \\ 0.7 & \text { if } k=0\end{array}\right.$. Thus, $\widetilde{F}$ is a fuzzy KMM-ideal of K.

Theorem 7. Order reversing holds true in every fuzzy KMMideal of $K$.

Proof. Let $k, m \in K$ be such that $k=m \Longrightarrow k \bullet m=0$. Now,

$$
\begin{aligned}
\widetilde{F}(k) & \geq \wedge\{\widetilde{F}(k \bullet m), \widetilde{F}(m)\} \\
& =\wedge\{\widetilde{F}(0), \widetilde{F}(m)\} \\
& =\widetilde{F}(m) .
\end{aligned}
$$

Thus, $\widetilde{F}$ is order reversing.

Theorem 8. Every fuzzy KM-subalgebra $\widetilde{F}$ is a fuzzy KMMideal of $K$.

Proof. Given $\widetilde{F}$ is a fuzzy KM-subalgebra of K.

$$
\begin{aligned}
\widetilde{F}(0) & =\widetilde{F}(k \bullet k) \\
& \geq \wedge\{\widetilde{F}(k), \widetilde{F}(k)\} \\
& =\widetilde{F}(k) .
\end{aligned}
$$

Therefore,

$$
\widetilde{F}(0) \geq \widetilde{F}(k) .
$$


And $\widetilde{F}(k) \geq \widetilde{F}(m)$ by Theorem 1

$$
\begin{aligned}
& =\wedge\{\widetilde{F}(0), \widetilde{F}(m)\} \operatorname{by}(1) \\
& =\wedge\{\widetilde{F}(k \bullet m), \widetilde{F}(m)\} .
\end{aligned}
$$

Thus, $\widetilde{F}(k) \geq \wedge\{\widetilde{F}(k \bullet m), \widetilde{F}(m)\}$.

Theorem 9. $\cap \widetilde{F}_{i}, i \in N$ of fuzzy KMM-ideals in $K$ is again a fuzzy KMM-ideal.

Proof. Let $k, m \in K$.

$$
\begin{aligned}
\left(\cap \widetilde{F}_{i}\right)(0) & =\wedge \bigwedge_{i \in N}\left(\widetilde{F}_{i}(0)\right) \geq \bigwedge_{i \in N}\left(\widetilde{F}_{i}(k)\right)=\cap \widetilde{F}_{i}(k) \\
\left(\cap \widetilde{F}_{i}\right)(k) & =\wedge_{i \in N}\left(\widetilde{F}_{i}(k)\right) \geq \wedge_{i \in N} \wedge\left\{\left(\widetilde{F}_{i}(k \bullet m)\right), \widetilde{F}_{i}(m)\right\} \\
& =\wedge\left\{\wedge_{i \in N}\left\{\left(\widetilde{F}_{i}(k \bullet m)\right), \inf \widetilde{F}_{i}(m)\right\}\right\} \\
& =\wedge\left\{\cap\left(\widetilde{F}_{i}(k \bullet m)\right), \cap \widetilde{F}_{i}(k)\right\} .
\end{aligned}
$$

$\cap \widetilde{F}_{i}$ is a fuzzy KMM-ideal.

\section{KM-Cartesian Product of Fuzzy KM-Algebras}

Here, an interesting notion $\mathrm{KM}$-Cartesian product in $\mathrm{KM}$ algebras has been introduced, and some of its properties have been analyzed.

Definition 6. Let $\widetilde{F}$ and $\widetilde{E}$ be the fuzzy KM-algebras. The fuzzy KM-Cartesian product $\widetilde{F} \otimes \widetilde{E}: K \times K \longrightarrow[0,1]$ is defined by $(\widetilde{F} \otimes \widetilde{E})(k, m)=\wedge\{\widetilde{F}(k), \widetilde{E}(m)\}$, for all $k, m \in K$. Here, the symbol $\otimes$ represents the operator of fuzzy KMCartesian product.
Theorem 10. $\widetilde{F} \otimes \widetilde{E}$ is a fuzzy KMM-ideal of $K \times K$ where $\widetilde{F}$ and $\widetilde{E}$ are fuzzy KMM-ideals of a KM-algebra K.

Proof. Let $(k, m) \in K \times K(\widetilde{F} \otimes \widetilde{E})(0,0)=\wedge\{\widetilde{F}(0), \widetilde{E}(0)\}$ by Definition 5 .

$\geq \wedge\{\widetilde{F}(k), \widetilde{E}(m)\}$ since $\widetilde{F}$ and $\widetilde{E}$ are fuzzy KMM-ideals of a KM-algebra K.

$$
=(\widetilde{F} \otimes \widetilde{E})(k, m) .
$$

Let $\left(k_{1}, m_{1}\right)$ and $\left(k_{2}, m_{2}\right) \in K \times K$.

$$
\begin{aligned}
(\widetilde{F} \otimes \widetilde{E})\left(k_{1}, k_{2}\right) & =\wedge\left\{\widetilde{F}\left(k_{1}\right), \widetilde{E}\left(k_{2}\right)\right\} \\
& \geq \wedge\left(\wedge\left\{\widetilde{F}\left(k_{1} \bullet m_{1}\right), \widetilde{F}\left(m_{1}\right)\right\},\right. \\
& \left.\wedge\left\{\widetilde{E}\left(k_{2} \bullet m_{2}\right), \widetilde{E}\left(m_{2}\right)\right\}\right) .
\end{aligned}
$$

Since $\widetilde{F}$ and $\widetilde{E}$ are fuzzy KMM-ideals of a KM-algebra K,

$$
\begin{aligned}
& =\wedge\left(\wedge\left\{\widetilde{F}\left(k_{1} \bullet m_{1}\right), \widetilde{E}\left(k_{2} \bullet m_{2}\right)\right\}, \wedge\left\{\widetilde{F}\left(m_{1}\right), \widetilde{E}\left(m_{2}\right)\right\}\right) \\
& =\wedge\left\{(\widetilde{F} \otimes \widetilde{E})\left(k_{1} \bullet m_{1}, k_{2} \bullet m_{2}\right),(\widetilde{F} \otimes \widetilde{E})\left(m_{1}, m_{2}\right)\right\} \text { by definition 5.1. } \\
& =\wedge\left\{\wedge\left\{\widetilde{F}\left(k_{1} \bullet m_{1}\right), \widetilde{E}\left(k_{2} \bullet m_{2}\right)\right\}, \wedge\left\{\widetilde{F}\left(m_{1}\right), \widetilde{E}\left(m_{2}\right)\right\}\right\} \\
& \geq \wedge\left\{\wedge\left\{\wedge\left\{\widetilde{F}\left(k_{1}\right), \widetilde{F}\left(m_{1}\right)\right\}, \wedge\left\{\widetilde{E}\left(k_{2}\right), \widetilde{E}\left(m_{2}\right)\right\}\right\}, \wedge\left\{\widetilde{F}\left(m_{1}\right), \widetilde{E}\left(m_{2}\right)\right\}\right\} \\
& =\wedge\left\{\wedge\left\{\wedge\left\{\widetilde{F}\left(k_{1}\right), \widetilde{E}\left(k_{2}\right)\right\}, \wedge\left\{\widetilde{F}\left(m_{1}\right), \widetilde{E}\left(m_{2}\right)\right\}\right\}, \wedge\left\{\widetilde{F}\left(m_{1}\right), \widetilde{E}\left(m_{2}\right)\right\}\right\} \\
& =\wedge\left\{\wedge\left\{\widetilde{F}\left(k_{1}\right), \widetilde{E}\left(k_{2}\right)\right\}, \wedge\left\{\widetilde{F}\left(m_{1}\right), \widetilde{E}\left(m_{2}\right)\right\}\right\} \\
& =\wedge\left\{(\widetilde{F} \otimes \widetilde{E})\left(k_{1}, k_{2}\right),(\widetilde{F} \otimes \widetilde{E})\left(m_{1}, m_{2}\right)\right\} \text { by definition 5.1. }
\end{aligned}
$$

Theorem 11. $\widetilde{F} \otimes \widetilde{E}$ is a fuzzy KM-subalgebra of $K \times K$ where $\widetilde{F}$ and $\widetilde{E}$ are fuzzy KM-subalgebras of a KM-algebra $K$.

Proof

$$
\begin{aligned}
{\left[(\widetilde{F} \otimes \widetilde{E})\left[\left(k_{1}, m_{1}\right) \otimes\left(k_{2}, m_{2}\right)\right]\right.} & =(\widetilde{F} \otimes \widetilde{E})\left(k_{1} \otimes k_{2}, m_{1} \otimes m_{2}\right) \\
& =\wedge\left\{\widetilde{F}\left(k_{1} \otimes k_{2}\right), \widetilde{E}\left(m_{1} \otimes m_{2}\right)\right\} \\
& \geq \wedge\left\{\wedge\left\{\widetilde{F}\left(k_{1}\right), \widetilde{F}\left(k_{2}\right)\right\}, \wedge\left\{\widetilde{E}\left(m_{1}\right), \widetilde{E}\left(m_{2}\right)\right\}\right\} \\
& =\wedge\left\{\wedge\left\{\widetilde{F}\left(k_{1}\right), \widetilde{E}\left(k_{2}\right)\right\}, \wedge\left\{\widetilde{F}\left(m_{1}\right), \widetilde{E}\left(m_{2}\right)\right\}\right\} \\
& =\wedge\left\{(\widetilde{F} \otimes \widetilde{E})\left(k_{1}, k_{2}\right),(\widetilde{F} \otimes \widetilde{E})\left(m_{1}, m_{2}\right)\right\} .
\end{aligned}
$$

Theorem 12. If $\widetilde{F}$ and $\widetilde{E}$ are fuzzy sets in a fuzzy KM-algebra $K$, where $\widetilde{F} \otimes \widetilde{E}$ is a fuzzy KMM-ideal of $K \times K$, then either $\widetilde{F}(0)=\widetilde{F}(k)$ or $\widetilde{E}(0)=\widetilde{E}(k)$, for all $k \in K$.

Proof. Assume that $\widetilde{F}(0)<\widetilde{F}(k)$ and $\widetilde{\mathrm{E}}(0)<\widetilde{E}(k)$ for some $k \in K$.

Then, for some

$$
\begin{aligned}
(\widetilde{F} \otimes \widetilde{E})(0 \times 0) & =\wedge\{\widetilde{F}(0), \widetilde{E}(0)\} \\
& <\wedge\{\widetilde{F}(k), \widetilde{E}(k)\} \\
& =(\widetilde{F} \otimes \widetilde{E})(k, k) .
\end{aligned}
$$

which is a contradiction to $\widetilde{F} \otimes \widetilde{E}$, a fuzzy KMM-ideals of $K \times K$. Hence proved.

Theorem 13. If $\widetilde{F} \otimes \widetilde{E}$ is a fuzzy KMM-ideal of $K \times K$ where $\widetilde{F}$ and $\widetilde{E}$ are fuzzy sets of a KM-algebra Kand $\widetilde{F}(0)=\widetilde{F}(k)$ for all $k \in K$, then either $\widetilde{E}(0)=\widetilde{F}(k)$ or $\widetilde{E}(0)=\widetilde{E}(k)$, for all $k \in K$.

Proof. Assume that $\widetilde{E}(0)<\widetilde{F}(k)$ and $\widetilde{E}(0)<\widetilde{E}(k)$ for some $k \in K$. 


$$
\begin{aligned}
(\widetilde{F} \otimes \widetilde{E})(0,0) & =\wedge\{\widetilde{F}(0), \widetilde{E}(0)\} \\
& =\widetilde{E}(0) \\
(\widetilde{F} \otimes \widetilde{E})(k, k) & =\wedge\{\widetilde{F}(k), \widetilde{E}(k)\} \\
& >\widetilde{E}(0) \\
& =(\widetilde{F} \otimes \widetilde{E})(0,0) \\
(\widetilde{F} \otimes \widetilde{E})(k, k) & >(\widetilde{F} \otimes \widetilde{E})(0,0) .
\end{aligned}
$$

which is a contradiction to $\widetilde{F} \otimes \widetilde{E}$, a fuzzy KMM-ideals of $K \times K$. Hence proved.

Similarly, we can prove $\widetilde{E}(0)=\widetilde{E}(k)$ for all $k \in K$, then either $\widetilde{F}(0)=\widetilde{F}(k)$ or $\widetilde{F}(0)=\widetilde{E}(k)$, for all $k \in K$.
Theorem 14. Let $\widetilde{F}$ and $\widetilde{E}$ be the fuzzy sets in a fuzzy KMalgebra $K$. If $\widetilde{F} \otimes \widetilde{E}$ is a fuzzy KMM-ideal of $K \times K$, then the fuzzy KMM-ideal of $K$ is $\widetilde{F}$ or $\widetilde{E}$.

Proof. By Theorem 12, we assume without losing generality that $\widetilde{E}(0) \geq \widetilde{E}(k)$ for all $k \in K$. By Theorem 13, either $\widetilde{F}(0) \geq \widetilde{F}(k)$ or $\widetilde{F}(0) \geq \widetilde{E}(k)$, for all $k \in K$.

If $\widetilde{F}(0) \geq \widetilde{E}(k)$, for all $k \in K$, then

$$
(\widetilde{F} \otimes \widetilde{E})(0, k)=\wedge\{\widetilde{F}(0), \widetilde{E}(k)\}=\widetilde{E}(k) .
$$

Given $\widetilde{F} \otimes \widetilde{E}$ is a fuzzy KMM-ideal of $K \times K$.

$$
\begin{aligned}
(\widetilde{F} \otimes \widetilde{E})\left(k_{1}, k_{2}\right) & \geq \wedge\left\{(\widetilde{F} \otimes \widetilde{E})\left(k_{1}, k_{2}\right) \bullet(\widetilde{F} \otimes \widetilde{E})\left(m_{1}, m_{2}\right),(\widetilde{F} \otimes \widetilde{E})\left(m_{1}, m_{2}\right)\right\} \\
& =\wedge\left\{(\widetilde{F} \otimes \widetilde{E})\left(k_{1} \bullet m_{1}\right),\left(k_{2} \bullet m_{2}\right),(\widetilde{F} \otimes \widetilde{E})\left(m_{1}, m_{2}\right)\right\} .
\end{aligned}
$$

Taking $k_{1}=m_{1}=0$, we have

$$
\begin{aligned}
(\widetilde{F} \otimes \widetilde{E})\left(0, k_{2}\right) & \geq \wedge\left\{(\widetilde{F} \otimes \widetilde{E})(0 \bullet 0),\left(k_{2} \bullet m_{2}\right),(\widetilde{F} \otimes \widetilde{E})\left(0, m_{2}\right)\right\} \\
\widetilde{E}\left(k_{2}\right) & \geq \wedge\left\{(\widetilde{F} \otimes \widetilde{E})(0),\left(k_{2} \bullet m_{2}\right),(\widetilde{F} \otimes \widetilde{E})\left(0, m_{2}\right)\right\} .
\end{aligned}
$$

By $\quad(22), \quad \widetilde{E}\left(k_{2}\right) \geq \wedge\left\{\widetilde{E}\left(k_{2} \bullet m_{2}\right), \widetilde{E}\left(m_{2}\right)\right\}$. Hence proved.

\section{Conclusion}

We have introduced the new notions of fuzzy product KMsubalgebra, fuzzy KMM-ideal, and fuzzy KM-Cartesian product. Also, we have proved some results like arbitrary intersection of fuzzy product KM-subalgebra is again a fuzzy KM-subalgebra and arbitrary intersection of fuzzy KMMideal is again a fuzzy KMM-ideal, and also some interesting results have been investigated, and it laid the foundation of our future research work. The definitions and key conclusions of fuzzy product KM-subalgebras, in our opinion, can be applied to a variety of different algebraic systems, particularly B-algebras such as BV-algebra, BF-algebra, BMalgebra, BN-algebra, BO-algebra, and BT-algebra.

(1) Our findings could potentially be used in engineering.

(2) Fuzzy product KM-subalgebras may be extended in the future to include fuzzy product KM-homomorphism and fuzzy product KM-ideals.

(3) Fuzzy product KM-subalgebras can indeed be extended in our future work

(1) To find the interval-valued fuzzy product KM-subalgebras.

(2) To find the intuitionistic fuzzy product KM-subalgebras.
(3) To find negative-valued fuzzy product KM-subalgebras.

(4) To establish more results in fuzzy product KM-subalgebras and application.

\section{Data Availability}

No data were used to support this study.

\section{Disclosure}

The statements made and views expressed are solely the responsibility of the author.

\section{Conflicts of Interest}

The authors declare that they have no conflicts of interest.

\section{Authors' Contributions}

All authors contributed equally and significantly in writing this article. All authors read and approved the final manuscript.

\section{Acknowledgments}

The sixth author (YUG) would like to acknowledge that this publication was made possible by a grant from Carnegie Corporation of New York. 


\section{References}

[1] M. A. A. Ansari and M. Chandramouleeswaran, "Fuzzy dot \$ \Beta-\$ subalgebras of \$ \$Beta-\$ algebras," International Journal of Pure and Applied Mathematics, vol. 90, no. 2, pp. 119-129, 2014.

[2] M. A. A. Ansari and M. Chandramouleeswaran, "Fuzzy dot $\beta$-ideals of $\beta$-algebras," International Journal of Mathematical Sciences and Engineering Applications, vol. 98, pp. 19-25, 2015.

[3] S. M. Hong, Y. B. Jun, S. J. Kim, and G. I. Kim, "On fuzzy dot subalgebras of BCH-algebras," International Journal of Mathematics and Mathematical Sciences, vol. 27, no. 6, pp. 357-364, 2001.

[4] Q. P. Hu, "On BCH-algebras," Mathematics Seminar Notes, vol. 11, pp. 313-320, 1983.

[5] I. H. Hwang, Y. L. Liu, and H. S. Kim, "On BV-Algebras," Mathematics, vol. 8, no. 10, p. 1779, 2020.

[6] Y. Imai and K. Iséki, "On axiom systems of propositional calculi. XIV," Proceedings of the Japan Academy, vol. 41, no. 6, pp. 436-439, 1966.

[7] K. Iséki, "An algebra related with a propositional calculus," Proceedings of the Japan Academy, vol. 42, no. 1, pp. 26-29, 1966.

[8] K. Kalaiarasi and V. Manimozhi, "Fuzzy sets in KM-algebras," Gedrag and Organisate Review, vol. 33, no. 2, pp. 2034-2046, 2020.

[9] C. B. Kim and H. S. Kim, "On BM-algebras," Scientiae Mathematicae Japonicae, vol. 63, no. 3, pp. 421-428, 2006.

[10] C. B. Kim and H. S. Kim, "On BG-algebras," Demonstratio Mathematica, vol. 41, no. 3, pp. 497-506, 2008.

[11] H. S. Kim and Y. H. Kim, "On BE-algebras," Scientiae Mathematicae Japonicae, vol. 66, no. 1, pp. 113-116, 2007.

[12] K. H. Kim, "On fuzzy dot subalgebras of d-algebras," International Mathematical Forum, vol. 4, no. 13, pp. 645-651, 2009.

[13] R. Kumar Bandaru, "On BRK-Algebras," International Journal of Mathematics \& Mathematical Sciences, vol. 2012, Article ID 952654, 2012.

[14] B. L. Meng, "CI-algebras," Scientiae Mathematicae Japonicae, vol. 71, no. 1, pp. 11-17, 2010.

[15] S. M. Mostafa and N. Cagman, "Fuzzy dot hyper KU-ideals," Journal of Hyperstructures, vol. 7, no. 2, 2019.

[16] S. M. Mostafa and R. A. Ghanem, "Fuzzy dot sub implicative ideals of KU-Algebras," Filomat, vol. 33, no. 6, 2019.

[17] J. Neggers and H. S. Kim, “On \$ d \$-algebras," Mathematica Slovaca, vol. 49, no. 1, pp. 19-26, 1999.

[18] J. Neggers and K. Sik, "On B-algebras," Matematièki vesnik, vol. 54, no. 1-2, pp. 21-29, 2002.

[19] J. Neggers, S. S. Ahn, and H. S. Kim, "On Q-algebras," International Journal of Mathematics and Mathematical Sciences, vol. 27, no. 12, pp. 749-757, 2001.

[20] A. B. Saeid, "Fuzzy dot BCK/BCI-algebras," International Journal of Algebra, vol. 4, no. 7, pp. 341-352, 2010.

[21] A. B. Saeid, H. S. Kim, and A. Rezaei, "On BI-algebras," "Analele Universitatii "Ovidius" "Constanta-Seria Matematica”, vol. 25, no. 1, pp. 177-194, 2017.

[22] P. S. Selvam and T. Priya, "Fuzzy dot Z-ideals of Z-algebras," Solid State Technology, vol. 64, no. 2, pp. 2363-2374, 2021.

[23] T. Senapati, M. Bhowmik, and M. Pal, "Fuzzy dot structure of BG-algebras," Fuzzy Information and Engineering, vol. 6, no. 3, pp. 315-329, 2014.
[24] T. Senapati, M. Bhowmik, and M. Pal, "Fuzzy dot subalgebras and fuzzy dot ideals of B-algebras," Journal of Uncertain Systems, vol. 8, no. 1, pp. 22-30, 2014.

[25] A. Walendziak, "On BF-algebras," Mathematica Slovaca, vol. 57, no. 2, pp. 119-128, 2007.

[26] L. A. Zadeh, "Fuzzy sets," Information and Control, vol. 8, pp. 338-353, 1965. 